\title{
A Survey of Patient Preferences for Oral Antihyperglycemic Therapy in Patients with Type 2 Diabetes Mellitus
}

\author{
A. Brett Hauber $\cdot$ Kaan Tunceli · Jui-Chen Yang • \\ Ira Gantz • Kimberly G. Brodovicz • Charles M. Alexander • \\ Michael J. Davies • Larry Radican
}

To view enhanced content go to www.diabetestherapy-open.com

Received: October 31, 2014 / Published online: January 14, 2015

(C) The Author(s) 2015. This article is published with open access at Springerlink.com

\section{ABSTRACT}

Introduction: Previous research has demonstrated a correlation among patient preferences, dosing burden, and medication nonadherence, a well-recognized challenge in type 2 diabetes mellitus (T2DM). The objective of this study was to elicit preferences for alternative dosing regimens for oral antihyperglycemic therapies among patients with T2DM and to quantify differences in dosing preferences among patients with different characteristics.

Methods: Preferences for dosing of oral antihyperglycemic drugs (OAD) were evaluated

Electronic supplementary material The online version of this article (doi:10.1007/s13300-015-0094-2) contains supplementary material, which is available to authorized users.

A. B. Hauber $(\square)$

RTI Health Solutions, 200 Park Offices Drive, PO Box 12194, Research Triangle Park, NC, USA e-mail: abhauber@rti.org

K. Tunceli · I. Gantz · K. G. Brodovicz ·

C. M. Alexander · M. J. Davies · L. Radican

Merck Sharp \& Dohme Corp., Whitehouse Station,

NJ, USA

J.-C. Yang

Pacific Economic Research, LLC, Bellevue, WA, USA by surveying patients with T2DM in the United States (US). Survey participants were adult US patients with T2DM who were taking no or only $1 \mathrm{OAD}$ and no injectable therapies. Each patient completed a web-enabled discrete-choice experiment (DCE) including a series of 8 pairs of hypothetical OAD profiles. Each profile was defined by reductions in average glucose, dosing schedule (e.g., once-weekly, once-daily, or twice-daily dosing), chance of mild-tomoderate gastrointestinal side effects, frequency of hypoglycemia, weight change, incremental risk of congestive heart failure, and cost. Each participant also answered a direct question about dosing preference. Random-parameters logit was used to analyze the DCE data. Prespecified subgroups were analyzed.

Results: Of 2,262 patients invited to participate, 923 were included in the analysis (mean age 63 years, 45\% male, 79\% white). Reducing dosing frequency was statistically significantly important to patients; however, it was relatively less important than medication cost or clinical outcomes. On average, patients preferred once-weekly to once-daily dosing. Patients not currently taking an OAD had a 
stronger preference for once-weekly dosing than patients on treatment $(P=0.012)$. Patients younger than 45 years had a stronger preference for weekly dosing than older patients $(P<0.075)$.

Conclusions: For younger patients and patients not currently on treatment, once-weekly dosing may provide additional incentive to initiate and adhere to antihyperglycemic treatment; however, additional research will be required to confirm this hypothesis.

Keywords: Conjoint analysis; Discrete-choice experiment; Patient preferences; Treatment adherence; Type 2 diabetes mellitus

\section{INTRODUCTION}

Previous research has demonstrated a correlation between dosing burden and medication nonadherence [1-3]. In type 2 diabetes mellitus (T2DM), therapies including combinations of oral antihyperglycemic drugs (OADs) can result in high nonadherence [4]. However, poor adherence to oral antidiabetes therapies is not limited to combination therapies and is common among patients just starting monotherapy [5]. A number of studies have demonstrated that patients with T2DM are willing to forgo the benefits of treatment to reduce treatment burden [6] and have identified a relationship between patients' preferences and likely medication adherence [7, 8]. Other studies have suggested that less frequent dosing may result in greater patient adherence, improved treatment outcomes, and reduced health care costs [9-12].

Discrete-choice experiments (DCEs), also known as choice-format conjoint analysis studies, increasingly have been used to determine the tradeoffs that patients are willing to make among features of medical interventions [13-15]. This method is based on the premise that medical interventions are composed of a set of attributes or outcomes, that the attractiveness of a particular intervention to an individual is a function of these attributes, and that choices among alternatives reveal patients' relative preferences for these attributes [16]. A recent study demonstrated that patients with T2DM have preferences for reducing the dosing burden of combination OAD therapy and that patients with lower current overall medication burden were more likely to indicate that decreases in dosing burden would result in an improvement in their expected medication adherence [7]. The objective of the current study was to elicit preferences for alternative dosing regimens for OAD therapies among patients with T2DM and to quantify differences in dosing preferences among patients with different characteristics.

\section{METHODS}

\section{Study Sample}

Patients were recruited from a probabilitybased, nationally representative panel of United States (US) households maintained by Knowledge Networks (Palo Alto, CA, USA). To qualify for inclusion in this study, patients had to (1) be aged 18 years or older; (2) have a selfreported physician diagnosis of T2DM; (3) be currently taking one $\mathrm{OAD}$ or no $\mathrm{OAD}$ to treat their T2DM; and (4) be not currently taking injectable T2DM treatments (e.g., insulin and glucagon-like-1 receptor agonist). Email invitations were sent to potential panelists based on self-reported physician diagnosis of T2DM. The sample was stratified by age (18-44, $45-64$, $\geq 65$ years), time since diagnosis $(\leq 3$, 
$>3$ years), and treatment status (no current $\mathrm{OAD}$, one current $\mathrm{OAD}$ ).

\section{Survey Instrument}

The online DCE survey was adapted from a survey used in a previous study [7] and developed using good research practices [13]. The survey included a series of eight choice questions; each question presented a pair of hypothetical, but realistic, OAD medication profiles (Fig. 1). Patients were asked to choose their preferred medication profile in each pair. Each profile was defined by five clinical outcomes, dosing schedule, and out-of-pocket cost. Clinical outcomes included reduction in average glucose (AG) [17] (between 20 and $66 \mathrm{mg} / \mathrm{dL}$ ) from a baseline of $206 \mathrm{mg} / \mathrm{dL}$, chance of mild-to-moderate gastrointestinal side effects (between 10\% and 30\%), frequency of hypoglycemia (between none and $>2$ hypoglycemic episodes per month), weight

Imagine your blood sugar level is 206 (uncontrolled). Please think about the following medicines, Medicine A and Medicine B.

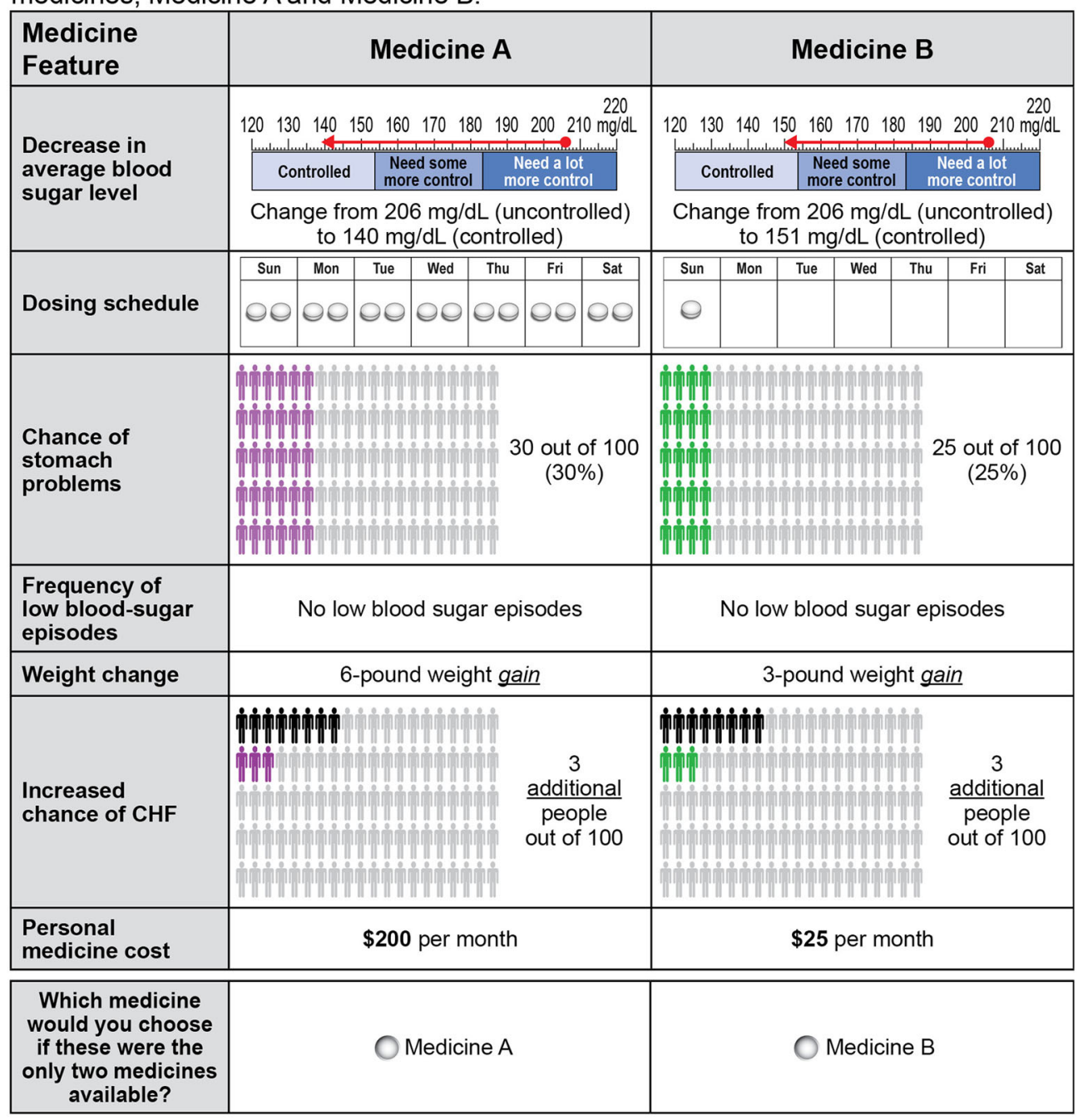

$\mathrm{CHF}=$ congestive heart failure.

Fig. 1 Example of a choice question in the discrete-choice experiment. $C H F$ congestive heart failure 
change within the first 6 months after starting treatment (between $6 \mathrm{lb}$ weight gain and $6 \mathrm{lb}$ weight loss), and incremental treatment-related risk of congestive heart failure (CHF) (between $0 \%$ and $3 \%$ ). The combination of attributes in each medication profile and the pairing of profiles in each choice question were determined by an experimental design with known statistical properties developed using good research practices [18].

Relevant clinical attributes and dosing options were identified based on a review of clinical literature, product labels, and ongoing clinical trials in the public domain for OADs, and in consultation with clinical experts [7]. The ranges of the attribute levels were chosen to encompass the range of outcome levels described in the clinical trials literature, as well as the range over which patients were willing to accept tradeoffs [7]. The dosing attribute included three levels (one pill once daily, two pills once daily, and one pill twice daily) based on available starting doses for common OADs prescribed to patients just starting monotherapy. The dosing attribute also included a once-weekly level to account for the future possibility of a once-weekly dipeptidyl peptidase-4 (DDP-4) inhibitor (ClinicalTrials.gov Identifier NCT01814748). Levels of out-of-pocket cost ranged from $\$ 0$ per month to $\$ 200$ per month and spanned the range over which the majority of patients were willing to trade among cost and other attributes in pretest interviews. The validity of the survey instrument was evaluated during 14 face-to-face qualitative pretest interviews with a convenience sample of patients with T2DM.

Following the choice questions, each patient was presented with a direct question about dosing preference (Fig. 2). Specifically, each patient was asked to indicate his or her preferred dosing schedule from four possible dosing schedules, assuming all other treatment characteristics were constant across alternatives.

The survey also collected data on demographic characteristics, health history, and treatment experience. The study and survey design were approved by Research Triangle Institute's Office of Research Protection and Ethics. All procedures followed were in accordance with the ethical standards of the responsible committee on human experimentation (institutional and national) and with the Helsinki Declaration of 1975, as revised in 2000 and 2008. All patients were required to provide informed consent before completing the survey. A copy of the survey instrument is available from the corresponding author upon request.

\section{Statistical Analysis}

The medication choice data were analyzed using a random-parameters logit model with NLOGIT 4.0 (Econometric Software, Inc, Plainview, NY, USA). Random-parameters logit controls for unobserved preference heterogeneity among respondents by estimating a distribution for each preference parameter $[19,20]$. The resulting parameter estimates can be interpreted as relative preference weights. The difference between the relative preference weights on the best and worst levels of the attribute is the relative importance of each attribute over the range of levels included in the survey.

The proportion of patients who preferred once-weekly dosing over daily-dosing alternatives was calculated for the overall sample and for each of the prespecified subgroups. For each pair of subgroups, $P$ values $(\alpha=0.05)$ were calculated using a Chi-squared 
If all oral diabetes medicines work the same and have the same side effects, which of the following dosing schedules would you choose? (Please select only one.)

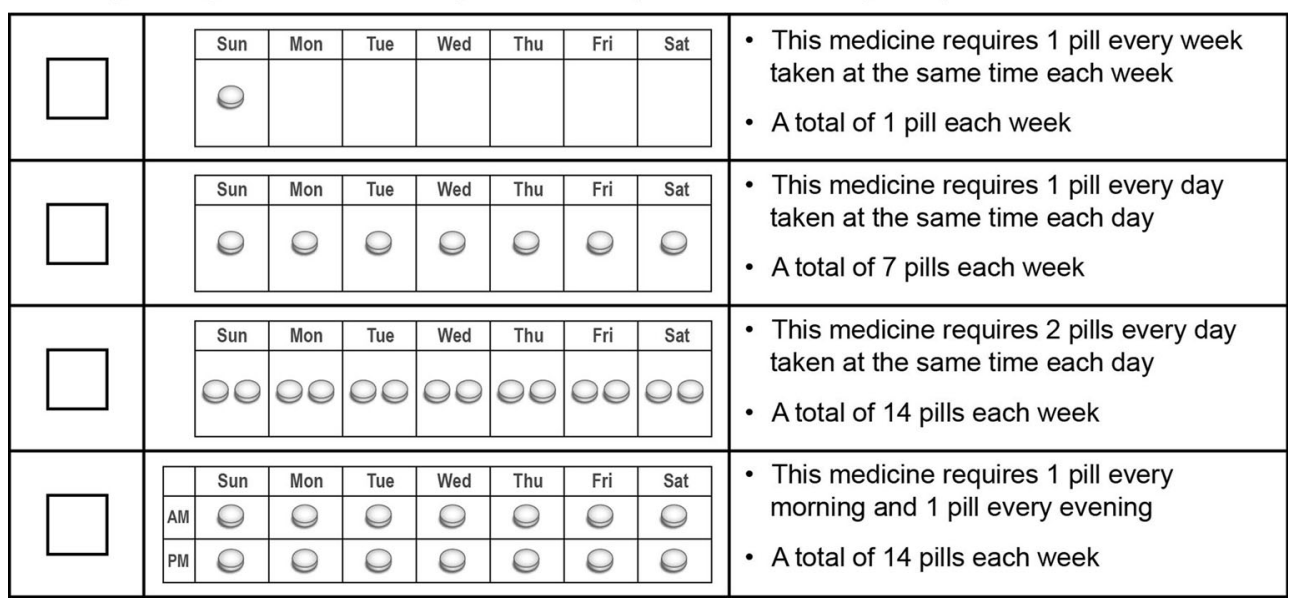

Fig. 2 Direct dosing question

test of the difference between the proportion of patients who preferred once-weekly dosing and the proportion of patients who preferred oncedaily dosing.

\section{RESULTS}

\section{Study Sample}

A total of 2,262 email invitations were sent to panel members. Of the 940 patients who responded and consented to participate, 13 patients were excluded from the analysis because they had no variation in their responses (i.e., they chose either Medicine A or Medicine B for every choice question). This response pattern indicated that the patient was inattentive to the choice questions. An additional 4 patients did not complete any of the choice questions. The final sample included 923 respondents with a mean age of 63 years; $45 \%$ of the respondents were male, and $79 \%$ were white. Most respondents (79\%) reported that they were currently using an OAD. Table 1 summarizes the characteristics of the respondents.

\section{Relative Importance Scores}

Relative importance scores are presented in Fig. 3. The distance between preference weights for the best level and worst level of an attribute can be interpreted as the overall relative importance of the attribute over the range of levels assessed in the survey. Over the ranges of levels included in the study, out-ofpocket cost (between $\$ 0$ and $\$ 200$ per month) was the most important treatment attribute and was statistically significantly more important than efficacy, side effects, and dosing. Reduction in AG was statistically significantly more important than dosing and the remaining clinical outcomes. Hypoglycemia, chance of mild-to-moderate gastrointestinal side effects, weight change within the first 6 months of starting treatment, and incremental increase in the risk of CHF were of approximately equal importance. Once-weekly dosing was preferred to one pill once daily. One pill once daily was preferred to one pill twice daily, which, in turn, was preferred to two pills once daily. The difference in preference weights between the most preferred dosing option (one pill once 
Table 1 Patient characteristics

\begin{tabular}{ll}
\hline Characteristics & $\begin{array}{l}\text { Patients } \\
(\boldsymbol{N}=\mathbf{9 2 3})\end{array}$ \\
\hline Male & $45 \%$ \\
White & $79 \%$ \\
Have health insurance ${ }^{\mathrm{a}}$ & $95 \%$ \\
Currently being treated with OADs & $79 \%$ \\
Mean age, years (SD) & $63(11)$ \\
Age subgroups & \\
$18-44$ years & $6.7 \%$ \\
$45-64$ years & $47.6 \%$ \\
$\geq 65$ years & $45.7 \%$ \\
Treatment status & \\
On treatment (currently taking an OAD) & 726 \\
Not on treatment (not currently & 197 \\
$\quad$ taking an OAD) & \\
Time since diagnosis & \\
$\leq 3$ years & \\
$>3$ years & 645 \\
\hline$O A D$ b
\end{tabular}

$O A D s$ oral antihyperglycemic drugs, T2DM type 2 diabetes mellitus, $S D$ standard deviation

${ }^{a}$ Six respondents with missing data

b Seven respondents did not report when they were first diagnosed with T2DM

weekly) and one pill once daily was positive and statistically significant; however, changes in dosing schedule were relatively less important than medication cost or changes in clinical outcomes over the ranges of attribute levels included in this study.

\section{Dosing Preferences}

Patients' dosing preferences are presented in Fig. 4. Among all patients, $67 \%$ preferred weekly dosing to any of the daily-dosing schedules. Among patients not currently taking any OAD, $75 \%$ preferred once-weekly dosing to daily- dosing options. This proportion was statistically significantly greater $(P=0.012)$ than the $65 \%$ of patients currently using an OAD who preferred once-weekly dosing over daily dosing. Among younger patients (aged $<45$ years), $78 \%$ preferred once-weekly dosing to daily dosing; in contrast, $66 \%$ of patients aged $45-64$ years $(P=0.065$ vs. the younger age group) and $66 \%$ of patients aged 65 years or older $(P=0.074$ vs. the younger age group) preferred once-weekly dosing to daily dosing, although these findings were not statistically significant. Similar proportions of patients whose T2DM was diagnosed within the previous 3 years and patients whose T2DM was diagnosed more than 3 years ago preferred once-weekly dosing to daily dosing: $66 \%$ of patients with a more recent diagnosis preferred once-weekly dosing, and $67 \%$ of patients with a diagnosis more than 3 years ago preferred onceweekly dosing.

\section{DISCUSSION}

Dosing burden has been found to influence patients' expectations about medication adherence in T2DM [7] and in other chronic diseases [21]. In this study, we administered a survey among patients with T2DM to elicit their preferences among treatment attributes (including efficacy, tolerability, daily dosing, and cost) to quantify the relative importance of reducing $\mathrm{OAD}$ dosing burden. Dosing preferences were elicited using both a DCE and a direct question.

Many studies have examined patients' preferences for dosing; however, the results of these studies are mixed. Some studies find that patients have statistically significant preferences for less frequent dosing while others do not. For example, Hauber et al. [7] 


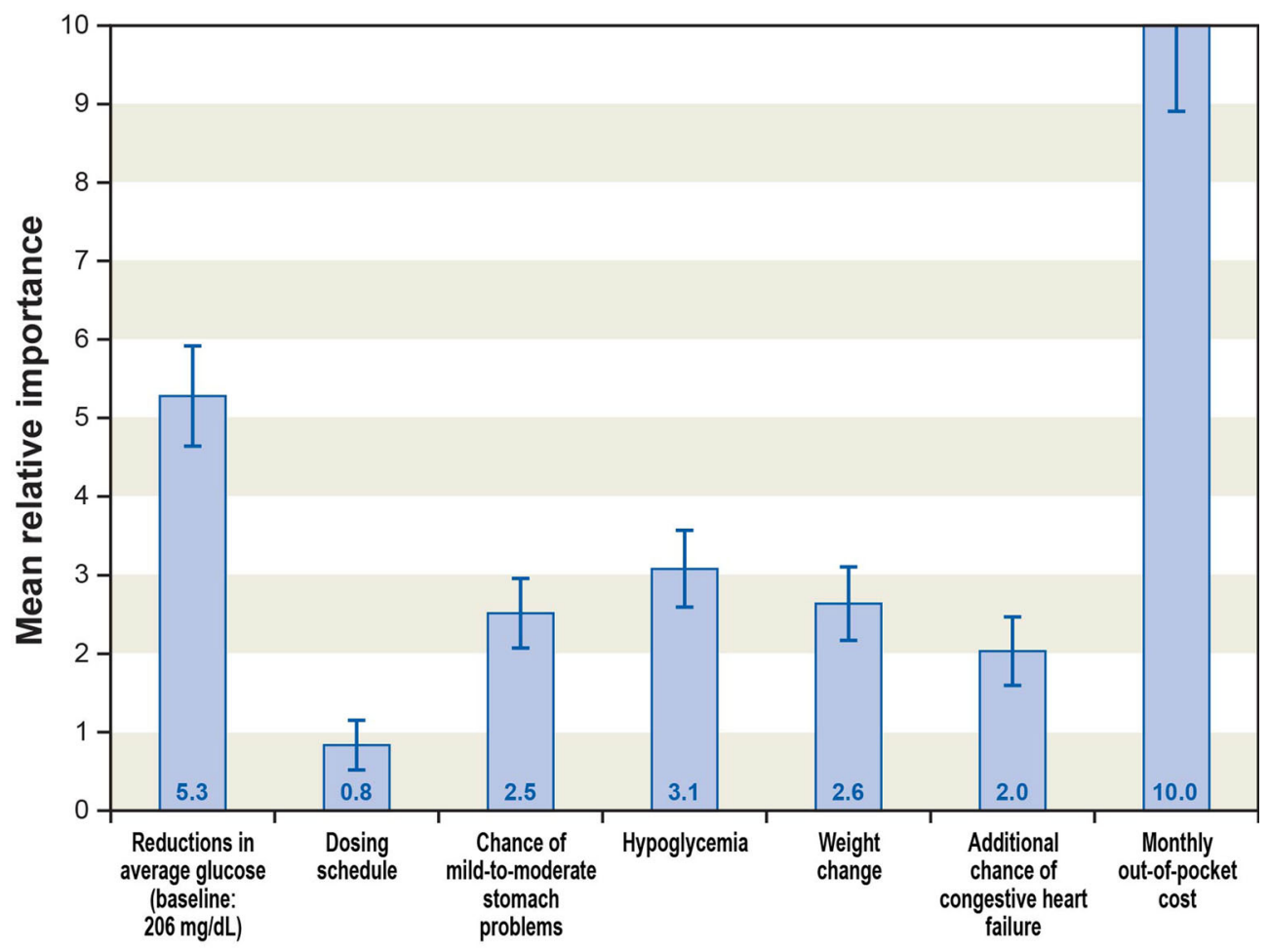

a The largest relative importance score was set equal to 10 and all other relative importance scores were calculated relative to the largest relative importance score.

Fig. 3 Relative importance scores for attributes in the discrete-choice experiment. The largest relative importance score was set equal to 10 and all other relative importance scores were calculated relative to the largest relative importance score

found that patients with T2DM had statistically significant preferences for reducing daily-dosing frequency, especially among patients with lower current dosing burdens. Likewise, de Bekker-Grob et al. [22] found that reducing the frequency of dosing for osteoporosis prophylaxis was approximately equally important as reducing the 10-year risk of osteoporosis by 10 percentage points among women in the Netherlands. In contrast, Lancsar et al. [23] and King et al. [24] found that preferences for reducing the frequency of daily dosing of asthma medications were not statistically significant predictors of treatment choice among asthma patients in Australia.

Although dosing was less important to patients with T2DM than clinical outcomes and cost in this study, patients had strong and statistically significant preferences for changes in dosing. In addition, patients had strong and statistically significant preferences for onceweekly dosing relative to alternative dailydosing regimens. Patients not currently taking an $\mathrm{OAD}$ had stronger preferences for weekly dosing than did patients who were currently taking an OAD. A higher proportion of patients aged 44 years and younger preferred onceweekly dosing to once-daily dosing when compared with patients aged 45 years and older, although this difference was not statistically significant. The lack of statistical significance for the difference in preferences for once-weekly dosing (12 percentage points) between younger and older patients may be due to the small sample size in the lower age group. Time since diagnosis did not have a 


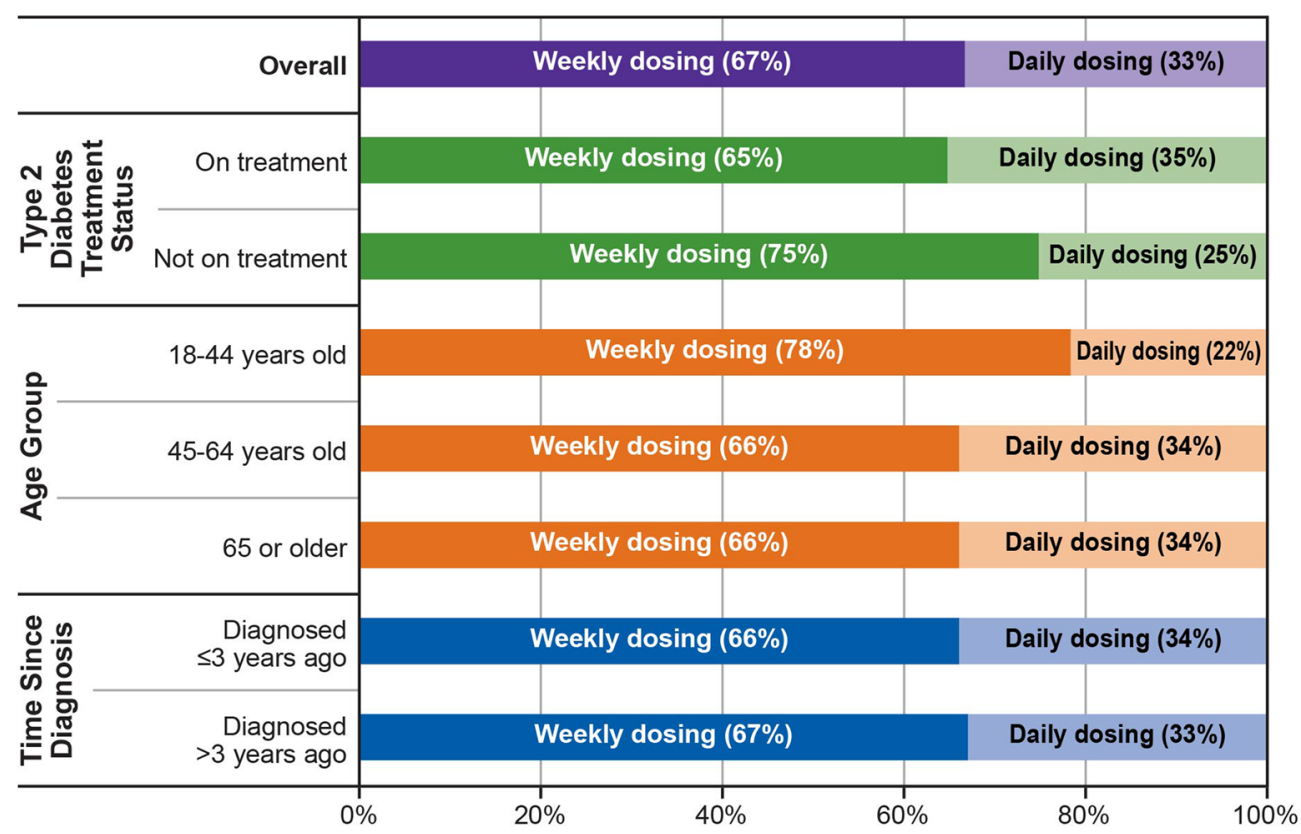

T2DM = type 2 diabetes mellitus.

Fig. 4 Proportion of patients preferring weekly dosing to daily dosing. All proportions are statically significantly different within the overall sample, within the subgroups, and across the subgroup pairings, except the following comparisons: respondents aged 18-44 years and respondents

measureable impact on dosing preferences among the patients in this study.

As all stated-preference studies, this study is subject to limitations. The results of this study should be interpreted with these limitations in mind. First, the survey asked patients to evaluate hypothetical scenarios. Although this practice is commonly used for eliciting patient preferences, the choices indicated by patients do not have the same impact as actual choices. In addition, none of the patients in this study had prior experience with weekly or less frequent dosing of OADs. Therefore, we cannot make any definitive statements about the effect of this type of experience on dosing preferences. Second, our study implicitly assumes that all clinical, convenience, and economic attributes of treatment that are not aged 45-64 years $\quad(P=0.065) ; \quad$ respondents aged $18-44$ years and respondents aged $\geq 65$ years $(P=0.074)$; and respondents whose T2DM was diagnosed $\leq 3$ years ago and respondents whose T2DM was diagnosed $>3$ years ago $(P=0.864) . T 2 D M$ type 2 diabetes mellitus

included in the DCE are held constant across the alternatives presented in the choice questions. In theory, adding these attributes to the DCE should not change our results; however, it is unknown whether including additional attributes in or deleting attributes from this particular study would yield different results. Finally, diagnosis of T2DM was selfreported, and the final sample may not be representative of the population of patients with T2DM in the US.

Patients in this study were asked to indicate which of four possible OAD dosing options they preferred. Overall, and in each subgroup evaluated in this study, patients preferred once-weekly dosing to daily dosing. These preferences were stronger for younger patients and patients not currently taking an OAD. 


\section{CONCLUSION}

All attributes used to describe T2DM medications were important to patients. Cost and glucose control had the greatest impact on treatment choice, indicating that although achieving treatment objectives is important to patients with T2DM, these patients may be willing to forgo optimal glucose control to avoid out-of-pocket costs. Dosing, tolerability, and adverse event risks were relatively less important than cost and glucose control; however, these treatment attributes were not unimportant in treatment choice. The majority of patients preferred once-weekly dosing to more frequent dosing. Patients not currently on treatment and younger patients (aged $<45$ years) were more likely to prefer once-weekly dosing to daily dosing. Therefore, for younger patients and patients not currently on treatment, once-weekly dosing may provide additional incentive to initiate and adhere to antihyperglycemic treatment. However, additional research will be required to confirm this hypothesis.

\section{ACKNOWLEDGMENTS}

Sponsorship for this study and article processing charges were funded by Merck Sharp \& Dohme Corp., Whitehouse Station, NJ USA. All authors meet the criteria for authorship as recommended by the International Committee for Medical Journal Editors, take responsibility for the integrity of the work as a whole, and have given final approval for the version to be published. Editorial assistance in the preparation of this manuscript was provided by Kate Lothman of RTI Health Solutions and was funded by Merck Sharp \& Dohme Corp.
Conflict of interest. A. Brett Hauber is an employee of RTI Health Solutions, a company sponsored by Merck Sharp \& Dohme Corp. to conduct this study and prepare the manuscript. Jui-Chen Yang was an employee of RTI Health Solutions during the conduct of this study but is no longer affiliated with RTI Health Solutions. Kaan Tunceli is an employee of Merck Sharp \& Dohme Corp. Ira Gantz is an employee of Merck Sharp \& Dohme Corp. Kimberly G. Brodovicz is an employee of Merck Sharp \& Dohme Corp. Charles M. Alexander is an employee of Merck Sharp \& Dohme Corp. Michael J. Davies is an employee of Merck Sharp \& Dohme Corp. Larry Radican is an employee of Merck Sharp \& Dohme Corp.

Compliance with ethics guidelines. The study and survey design were approved by Research Triangle Institute's Office of Research Protection and Ethics. All procedures followed were in accordance with the ethical standards of the responsible committee on human experimentation (institutional and national) and with the Helsinki Declaration of 1975, as revised in 2000 and 2008. Informed consent was obtained from all patients for being included in the study. All patients were required to provide informed consent before completing the survey.

Open Access. This article is distributed under the terms of the Creative Commons Attribution Noncommercial License which permits any noncommercial use, distribution, and reproduction in any medium, provided the original author(s) and the source are credited.

\section{REFERENCES}

1. Coleman CI, Limone B, Sobieraj DM, et al. Dosing frequency and medication adherence in chronic disease. J Manag Care Pharm. 2012;18:527-39. 
2. García-Perez L, Alvarez M, Dilla T, Gil-Guillén V, Orozco-Beltrán D. Adherence to therapies in patients with type 2 diabetes. Diabetes Ther. 2013;4:175-94.

3. Srivastava K, Arora A, Kataria A, Cappelleri JC, Sadosky A, Peterson AM. Impact of reducing dosing frequency on adherence to oral therapies: a literature review and meta-analysis. Patient Prefer Adherence. 2013;7:419-34.

4. Mateo JF, Gil-Guillen VF, Mateo E, Orozco D, Carbayo JA, Merino J. Multifactorial approach and adherence to prescribed oral medications in patients with type 2 diabetes. Int J Clin Pract. 2006;60:422-8.

5. Shenolikar RA, Balkrishnan R. Oral antidiabetes medication adherence and health care utilization among Medicaid-enrolled type 2 diabetic patients beginning monotherapy. Diabetes Care. 2008;31:e5.

6. Hauber AB, Johnson FR, Sauriol L, Lescrauwaet B. Risking health to avoid injections: preferences of Canadians with type 2 diabetes. Diabetes Care. 2005;28:2243-5.

7. Hauber AB, Han S, Yang J-C, Gantz I, Tunceli K, Gonzalez JM, et al. Effect of pill burden on dosing preferences, willingness to pay, and likely adherence among patients with type 2 diabetes. Patient Prefer Adherence. 2013;2013:937-49.

8. Hauber AB, Mohamed AF, Johnson FR, Falvey H. Treatment preferences and medication adherence of people with Type 2 diabetes using oral glucoselowering agents. Diabet Med. 2009;26:416-24.

9. Bae JP, Dobesh PP, Klepser DG, et al. Adherence and dosing frequency of common medications for cardiovascular patients. Am J Manag Care. 2012;18:139-46.

10. Claxton AJ, Cramer J, Pierce C. A systematic review of the associations between dose regimens and medication compliance. Clin Ther. 2001;23:1296-310.

11. Richter A, Anton SE, Koch P, Dennett SL. The impact of reducing dose frequency on health outcomes. Clin Ther. 2003;25:2307-35.

12. Saini SD, Schoenfeld P, Kaulback K, Dubinsky MC. Effect of medication dosing frequency on adherence in chronic diseases. Am J Manag Care. 2009;15:e22-33.

13. Bridges JFP, Hauber AB, Marshall D, Lloyd A, Prosser LA, Regier DA, et al. Conjoint analysis applications in health-a checklist: a report of the ISPOR Good Research Practices for Conjoint Analysis Task Force. Value Health. 2011;14:403-13.

14. Marshall D, Bridges JFP, Hauber AB, et al. Conjoint analysis applications in health-how are studies being designed and reported? An update on current practice in the published literature between 2005 and 2008. Patient. 2010;3:249-56.

15. de Bekker-Grob EW, Ryan M, Gerard K. Discrete choice experiments in health economics: a review of the literature. Health Econ. 2012;21:145-72.

16. Hensher DA, Rose JM, Greene WH. Applied choice analysis. Cambridge: Cambridge University Press; 2005.

17. Nathan DM, Kuenen J, Borg R, A1c-Derived Average Glucose Study Group, et al. Translating the A1C assay into estimated average glucose values. Diabetes Care. 2008;31(8):1473-8.

18. Johnson FR, Lancsar E, Marshall D, et al. Constructing experimental designs for discretechoice experiments: report of the ISPOR Conjoint Analysis Experimental Design Good Research Practices Task Force. Value Health. 2013;16:3-13.

19. Train K, Sonnier G. Mixed logit with bounded distributions of correlated partworths. In: Scarpa R, Alberini A, editors. Applications of simulation methods in environmental and resource economics. Dordrecht: Springer; 2005.

20. Train K. Discrete choice methods with simulation. Cambridge: Cambridge University Press; 2003.

21. Laba TL, Essue B, Kimman M, Jan S. Understanding patient preferences in medication nonadherence: a review of stated preference data. Patient. 2014. doi:10.1007/s40271-014-0099-3.

22. de Bekker-Grob EW, Essink-Bot ML, Meerding WJ, et al. Patients' preferences for osteoporosis drug treatment: a discrete choice experiment. Osteoporos Int. 2008;19:1029-37.

23. Lancsar EJ, Hall JP, King M, Kenny P, et al. Using discrete choice experiments to investigate subject preferences for preventive asthma medication. Respirology. 2007;12:127-36.

24. King MT, Hall J, Lancsar E, et al. Patient preferences for managing asthma: results from a discrete choice experiment. Health Econ. 2007;16:703-17. 